\title{
High-temperature magnetism and microstructure of a semiconducting ferromagnetic $(\mathrm{GaSb})_{1-x}(\mathrm{MnSb})_{x}$ alloy
}

\author{
Leonid N. Oveshnikov ${ }^{* 1,2}$, Elena I. Nekhaeva ${ }^{1,2}$, Alexey V. Kochura ${ }^{3}$, \\ Alexander B. Davydov2 ${ }^{2}$, Mikhail A. Shakhov ${ }^{4,5}$, Sergey F. Marenkin ${ }^{6,7}$, \\ Oleg A. Novodvorskii ${ }^{8}$, Alexander P. Kuzmenko ${ }^{3}$, Alexander L. Vasiliev ${ }^{1}$, \\ Boris A. Aronzon ${ }^{1,2}$ and Erkki Lahderanta ${ }^{4}$
}

\section{Full Research Paper}

\section{Address:}

${ }^{1}$ National Research Center "Kurchatov Institute", 123182 Moscow, Russian Federation, ${ }^{2}$ P.N. Lebedev Physical Institute, Russian Academy of Sciences, 119991 Moscow, Russian Federation, ${ }^{3}$ South-West State University, 305040 Kursk, Russian Federation, ${ }^{4}$ Lappeenranta University of Technology, 53850 Lappeenranta, Finland, ${ }^{5}$ Ioffe Institute, 194021 St. Petersburg, Russian Federation, ${ }^{6}$ Kurnakov Institute of General and Inorganic Chemistry, Russian Academy of Sciences, 119991 Moscow, Russian Federation,

${ }^{7}$ National University of Science and Technology MISIS, 119049 Moscow, Russian Federation and ${ }^{8}$ Institute on Laser and Information Technologies, Russian Academy of Sciences, 140700 Shatura, Moscow Region, Russian Federation

Email:

Leonid N. Oveshnikov* - oveshln@gmail.com

* Corresponding author

Keywords:

anomalous Hall effect; high-temperature ferromagnetism; nanostructured materials; thin films

\begin{abstract}
We have studied the properties of relatively thick (about $120 \mathrm{~nm}$ ) magnetic composite films grown by pulsed laser deposition using the eutectic compound $(\mathrm{GaSb})_{0.59}(\mathrm{MnSb})_{0.41}$ as target for sputtering. For the studied films we have observed ferromagnetism and an anomalous Hall effect above room temperature, confirming the presence of spin-polarized carriers. Electron microscopy, atomic and magnetic force microscopy results suggest that the films under study have a homogenous columnar structure in the bulk while $\mathrm{MnSb}$ inclusions accumulate near the surface. This is in good agreement with the high mobility values of charge carriers. Based on our data we conclude that the magnetic and magnetotransport properties of the films at room temperature are defined by the MnSb inclusions.
\end{abstract}

Beilstein J. Nanotechnol. 2018, 9, 2457-2465.

doi:10.3762/bjnano.9.230

Received: 14 March 2018

Accepted: 25 August 2018

Published: 14 September 2018

Associate Editor: P. Leiderer

(c) 2018 Oveshnikov et al.; licensee Beilstein-Institut. License and terms: see end of document. 


\section{Introduction}

Diluted magnetic semiconductors (DMS) are very promising materials for spintronic devices, because DMS offer the combination of magnetic and semiconducting properties. Currently, the most commonly studied DMS systems are those based on III-V semiconductors doped by Mn [1-3]. Among these systems, the most well-known and extensively studied is $\mathrm{Ga}_{1-x} \mathrm{Mn}_{x} \mathrm{As}$. Here Mn atoms substitute $\mathrm{Ga}$ atoms and establish a ferromagnetic state realized through carrier-induced indirect exchange between Mn atoms by a Zener-RKKY mechanism accompanied by the spin polarization of conducting holes $[1,3]$ To reach high values of the Curie temperature, $T_{\mathrm{c}}$, materials with high Mn concentration are required, which can be achieved by using non-equilibrium growth methods, such as low-temperature molecular-beam epitaxy. Thus, the solubility limit of $\mathrm{Mn}$ in $\mathrm{Ga}_{1-x} \mathrm{Mn}_{x} \mathrm{As}$ can be increased up to $x=0.2$ without precipitate formation [4]. The highest $T_{\mathrm{c}}$ values achieved in these materials was below $200 \mathrm{~K}$, observed with $x$ values of about 0.1 $[5,6]$. This is remarkably high for a DMS system while for practical applications $T_{\mathrm{c}}>300 \mathrm{~K}$ is desired. At higher concentrations $\mathrm{Mn}$ atoms start to occupy interstitial sites and produce strong structure defects that increase the scattering of charge carriers. Thus, hole mobilities in $\mathrm{Ga}_{1-x} \mathrm{Mn}_{x} \mathrm{As}$ systems with high $T_{\mathrm{c}}(x>0.06)$ usually do not exceed $10 \mathrm{~cm}^{2} /(\mathrm{V} \cdot \mathrm{s})$.

Another way to realize high $T_{\mathrm{c}}$ values in magnetic semiconductor materials is to create a granular system with two phases, i.e., ferromagnetic nanoinclusions embedded into a semiconductor matrix. Although such systems are studied less frequently, in some of the related works the observed $T_{\mathrm{c}}$ values exceeded room temperature [7-13]. An additional advantage of granular systems are the higher values of carrier mobility, about one order of magnitude higher than that in traditional DMS such as $\mathrm{Ga}_{1-x} \mathrm{Mn}_{x} \mathrm{As}$. This is due to the aggregation of the majority of magnetic impurity atoms within nanoinclusions, which results in a higher crystalline quality of the semiconductor matrix [9] and a lower density of scatterers. Thus, granular materials could be of interest, both as an object of fundamental studies of DMS systems and as a versatile material suitable for testing prototype spintronic devices.

Recent studies of MnAs inclusions embedded into a GaAs matrix showed that inclusions can emerge with two types of crystal structure. The magnetic properties of MnAs inclusions with zinc blende type and hexagonal lattices are substantially different. The actual application of GaAs:MnAs materials is restricted by this fact.

A second recently studied nanocomposite system is a $\mathrm{GaSb}$ matrix with incorporated MnSb nanograins [8-10,14]. However, the best results were obtained not for a composite system but for $(\mathrm{GaSb})_{1-x}(\mathrm{MnSb})_{x}$ alloys with $x=0.41$. In annealed samples the mobility of holes was about $100 \mathrm{~cm}^{2} /(\mathrm{V} \cdot \mathrm{s})$ and $T_{\mathrm{c}}$ was above room temperature [11,12]. Earlier it was suggested [9] that the ferromagnetic ordering in this case is induced by the interaction of $\mathrm{MnSb}$ magnetic clusters with carriers inside the matrix. It should induce carrier spin-polarization and lead to the formation of a long-range ferromagnetic percolation cluster, which includes both MnSb magnetic clusters and spinpolarized carriers. However, to verify this assumption and to reveal the origin of the high-temperature ferromagnetism in $\mathrm{GaSb}-\mathrm{MnSb}$ alloys, one needs a detailed knowledge of the sample structure.

Thus, in this paper we investigate magnetic and transport properties of the GaSb-MnSb alloy films with $x=0.41$ and we elucidate the origin of the ferromagnetic state in this material. We use atomic force and magnetic force microscopy (AFM and MFM) as well as scanning/transmission electron microscopy (S/TEM) to study the sample structure.

\section{Experimental}

$\mathrm{GaSb}-\mathrm{MnSb}$ films with a thickness $d$ in the range between 120 and $135 \mathrm{~nm}$ and an area of $0.1-1.0 \mathrm{~cm}^{2}$ were grown by dropletfree pulsed laser deposition (PLD) in high vacuum $\left(10^{-6}\right.$ Torr $)$ with deposition temperatures of $T_{\mathrm{dep}}=100-300^{\circ} \mathrm{C}$. We employed a $\mathrm{GaSb}-\mathrm{MnSb}$ target of eutectic composition containing $41 \mathrm{~mol} \% \mathrm{MnSb}$ and $59 \mathrm{~mol} \% \mathrm{GaSb}$, which was sputtered by the second harmonic radiation of a Q-switched YAG:Nd laser $(\lambda=532 \mathrm{~nm}) . \mathrm{Al}_{2} \mathrm{O}_{3}(0001)$ single crystals were used as substrates. A more detailed description of the growth technology can be found in [15].

Magnetization was measured at temperatures of $T=5-310 \mathrm{~K}$ in magnetic fields up to $H=50 \mathrm{kOe}$ using a superconducting quantum interference device (SQUID) magnetometer S600X (Cryogenic, UK). The electrical and magnetotransport properties were investigated at temperatures of $T=2-320 \mathrm{~K}$ using a standard six-probe geometry in pulsed magnetic fields up to $H=300 \mathrm{kOe}$. The studied samples demonstrated linear current-voltage characteristics down to sub-helium temperatures while sustaining high values of conductivity.

The cross-section specimens for S/TEM studies were prepared by focus ion beam (FIB) milling in a Helios (FEI, US) SEM/FIB dual-beam system equipped with $\mathrm{C}$ and $\mathrm{Pt}$ gas injectors and a micromanipulator (Omniprobe, US). A $2 \mu \mathrm{m}$ Pt layer was deposited on the surface of the sample prior to the crosssection preparation by FIB milling. Sections of approximately $8 \times 5 \mu^{2}$ area and $2 \mu \mathrm{m}$ thickness were cut by $30 \mathrm{kV} \mathrm{Ga}^{+}$ions, 
removed from the sample and then attached to the Omniprobe semiring (Omniprobe, US). Final thinning was performed with $5 \mathrm{kV} \mathrm{Ga}^{+}$ions followed by cleaning by $2 \mathrm{keV} \mathrm{Ga}^{+}$ions for electron transparency. All specimens were studied in a scanning/ transmission electron microscope Titan 80-300 (FEI, US) equipped with a spherical aberration (Cs) corrector (electron probe corrector), a high-angle annular dark field (HAADF) detector, an atmospheric thin-window energy dispersive X-ray (EDX) spectrometer (Phoenix System, EDAX, US) and a postcolumn Gatan energy filter (GIF), (Gatan, US). The S/TEM was operated at $300 \mathrm{kV}$. Digital micrograph (Gatan, US) and TIA software (FEI, US) was used for image analysis. P. Stadelmann's JEMS software [16] was used for the simulation of diffraction patterns and images.

Scanning atomic force microscopy (AFM) and magnetic force microscopy (MFM) images were obtained on an SmartSPM microscope (AIST-NT, US) at temperatures of $T=295-450 \mathrm{~K}$.

\section{Results and Discussion}

We have studied several $(\mathrm{GaSb})_{1-x}(\mathrm{MnSb})_{x}$ samples with $x=0.41$, both annealed and not annealed. In this paper we discuss only samples annealed at $350{ }^{\circ} \mathrm{C}$ for $30 \mathrm{~min}$ with high hole concentrations of $N_{\mathrm{p}}>10^{19} \mathrm{~cm}^{-3}$ because they showed much better magnetic and semiconducting (electron transport) properties. Therefore, these samples are more suitable to reveal the nature of magnetic properties and hole spin-polarization in this material, which is the aim of this paper. Main parameters of the studied samples are presented in Table 1.

Figure 1a shows typical curves of the magnetization as function of the magnetic field for the sample GM3 (see Table 1) for magnetic field orientations parallel to the sample plane and perpendicularly to it. The presence of a well-pronounced hysteresis suggests that ferromagnetic ordering in these materials appears at $T>300 \mathrm{~K}$. This is more evident from the temperature dependence of the remanent magnetization $M_{\text {rem }}$ presented in

\begin{tabular}{|c|c|c|c|c|c|c|c|}
\hline sample & $\begin{array}{l}T_{\text {dep }} \\
{ }^{\circ} \mathrm{C}\end{array}$ & $\begin{array}{l}d, \\
\mathrm{~nm}\end{array}$ & $\begin{array}{l}N_{\mathrm{p}} \\
10^{19} \mathrm{~cm}^{-3}\end{array}$ & $\begin{array}{l}\mu, \\
\mathrm{cm}^{2} /(V \cdot s)\end{array}$ & $\begin{array}{l}H_{\mathrm{c}}^{\mathrm{a}} \\
\mathrm{Oe}\end{array}$ & $M_{\text {rem }}{ }^{a}$ & $M_{\text {sat }}{ }^{\mathrm{a}}$ \\
\hline GM1 & 100 & 120 & 0.8 & 110 & 250 & $1.4 \mu_{\mathrm{B}}$ & $1.8 \mu_{\mathrm{B}}$ \\
\hline GM2 & 100 & 120 & 9.3 & 110 & 260 & $2.1 \mu_{\mathrm{B}}$ & $3.0 \mu_{\mathrm{B}}$ \\
\hline GM3 & 200 & 130 & 23 & 80 & 260 & $1.7 \mu_{\mathrm{B}}$ & $3.0 \mu_{\mathrm{B}}$ \\
\hline GM4 & 200 & 135 & 14 & 71 & 115 & $2.1 \mu_{\mathrm{B}}$ & $3.2 \mu_{\mathrm{B}}$ \\
\hline GM5 & 300 & 120 & 4.8 & 74 & 265 & $0.6 \mu_{\mathrm{B}}$ & $2.5 \mu_{B}$ \\
\hline
\end{tabular}

aMagnetic parameters were obtained for magnetic field oriented parallel to the sample plane; the magnetization values were calculated per manganese atom.
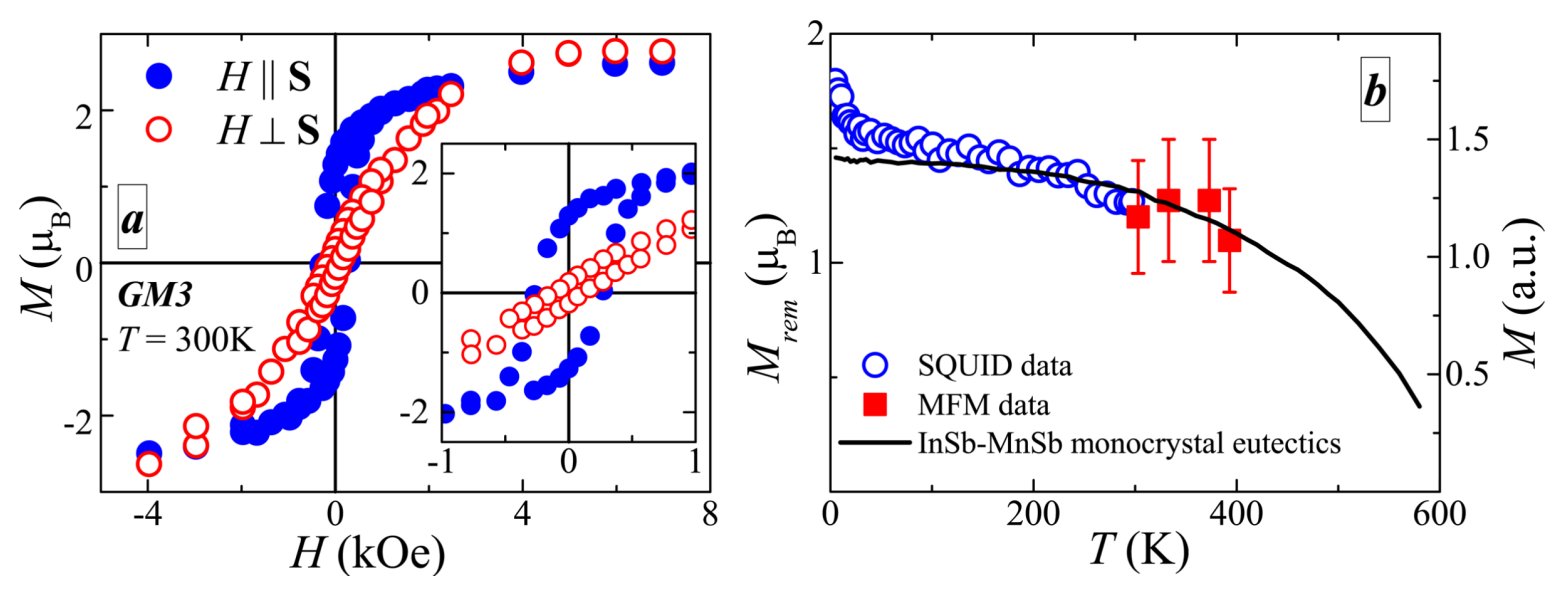

Figure 1: (a) Magnetization as a function of the magnetic field for sample GM3 at $T=300 \mathrm{~K}$. Measurements were performed with the magnetic field oriented parallel to the sample plane (solid symbols) and perpendicularly to it (open symbols). The inset shows the hysteresis loop at low fields. (b) Temperature dependence of the remanent magnetization. Open circles are SQUID data for sample GM3, red squares are MFM data for the same sample and the black curve shows the temperature dependence of the saturation magnetization of the $\operatorname{lnSb}-\mathrm{MnSb}$ eutectic [17]. 
Figure 1b. In the same figure we also provide the MFM data and temperature dependence of the remanent magnetization for InSb-MnSb eutectics. Nonzero $M_{\text {rem values persist up to } 400 \mathrm{~K}}$ and the temperature dependence is close to that of the InSb-MnSb eutectic composition, which corresponds to the $T_{\mathrm{c}}$ value of $\mathrm{MnSb}$ of ca. $600 \mathrm{~K}$ [17]. Thus, presented data clearly proves the presence of high-temperature ferromagnetism in the samples under study.

As mentioned above, the studied system differs from traditional DMS materials by substantially higher carrier mobilities and $T_{\mathrm{c}}$ values, while the nature of the high-temperature ferromagnetism is not completely clear. Previous studies [9] suggested that ferromagnetism in $(\mathrm{GaSb})_{1-x}(\mathrm{MnSb})_{x}$ is related to the interaction of charge carriers with $\mathrm{MnSb}$ nanoinclusions with $T_{\mathrm{c}}=600 \mathrm{~K}[17,18]$. This interaction is affected by the appearance of Schottky barriers at the MnSb/GaSb boundaries. Basically, these type of barriers appear on the semiconductor/metal interfaces providing a tunneling charge transfer across the boundary, if the barrier is high enough. In the present case, Schottky barriers may appear only if inclusions are sufficiently large to establish a second electronic phase (metal-type) despite the impact of the surrounding GaSb matrix. The parameters of these barriers (e.g., the width) substantially depend on the charge distribution, i.e., they depend on the carrier concentration.

It is worth mentioning that in real samples not all $\mathrm{Mn}$ atoms are strictly positioned in $\mathrm{MnSb}$ inclusions, some of the Mn atoms can be distributed within the semiconductor matrix. This results in a two-phase magnetic subsystem in which lower $T_{\mathrm{c}}$ values correspond to isolated $\mathrm{Mn}$ atoms within the matrix [19]. Also, as it is shown in Table 1 , the $M_{\text {sat }}$ values reach $3.2 \mu_{\mathrm{B}}$ per Mn atom, which is close to $3.6 \mu_{\mathrm{B}}$ obtained from the experimental data for MnSb samples analyzed earlier [18,20], but is lower than the value expected for $\mathrm{Mn}^{2+}$. This difference can be related to the presence of $\mathrm{Mn}^{3+}$ ions and antiferromagnetic interactions between carriers and $\mathrm{Mn}$ atoms.

One major parameter of the interaction of holes with $\mathrm{MnSb}$ inclusions is the width of the Schottky barriers $d_{\text {barrier, }}$, which decreases with increasing carrier concentration [9]. This picture correlates with the concentration dependence of the saturation magnetization $M_{\text {sat }}$ presented in Figure 2. This also agrees well with the data obtained for previously studied $(\mathrm{GaSb})_{1-x}(\mathrm{MnSb})_{x}$ films (see Figure 5 in [12]). As it can be seen from these figures, $M_{\text {sat }}$ increases with carrier concentration and saturates above $N_{\mathrm{p}} \approx 10^{20} \mathrm{~cm}^{-3}$ at which $d_{\text {barrier }}$ becomes comparable with the effective penetration depth of the carrier (hole) wave function, $l_{\mathrm{p}}$, under triangular barrier. Taking into account that the energy gap in $\mathrm{GaSb}$ is $E_{\mathrm{g}}=0.7 \mathrm{eV}$ and the Schottky barrier height is about (1/3) $E_{\mathrm{g}}[21]$, the value of $d_{\text {barrier }}$ can be estimated as $2 \mathrm{~nm}$ at $N_{\mathrm{p}}=10^{20} \mathrm{~cm}^{-3}$ while $l_{\mathrm{p}}$ is of the same value [9].

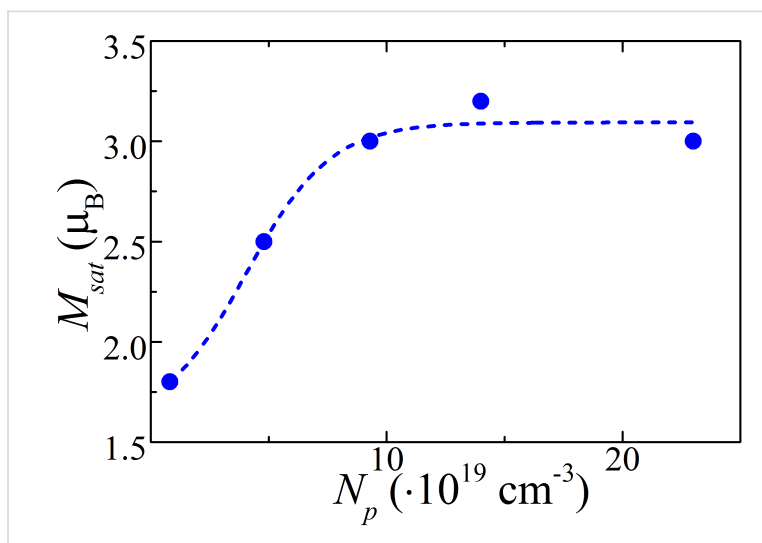

Figure 2: Room-temperature saturation magnetization $\left(M_{\text {sat }}\right)$ as a function of the carrier concentration $\left(N_{p}\right)$ (the dashed line is a guide to the eye).

As shown in Figure 2, the values of $N_{\mathrm{p}}$ for the studied samples are sufficiently high to provide an effective interaction of holes with the MnSb inclusions and cause the saturation of magnetization. This, along with high $T_{\mathrm{c}}$ values, suggests that this interaction could be the main source of the high-temperature ferromagnetism in the studied systems. However, to justify the applicability of a model of interaction between carriers and inclusions, one needs to verify specific properties of spin-polarized system, for instance through transport measurements.

It should be noted that the mobility values have no pronounced dependence on the carrier concentration (see Table 1), i.e., the conductivity of the studied films is affected by various factors. Thus, magnetotransport phenomena can yield additional information about the system. The results of Hall resistivity measurements for the GM3 sample are shown in Figure $3 a$ and Figure 3 b. The Hall resistivity in magnetic systems can be divided into two parts:

$$
R_{x y} \cdot d=R_{\mathrm{H}} \mathbf{H}+R_{\mathrm{s}} \mathbf{M}
$$

where $R_{\mathrm{H}}$ is the Hall constant, used for the determination of $N_{\mathrm{p}}$ and $\mu$ (see Table 1 ), and $R_{\mathrm{S}}$ is the anomalous Hall constant, which is defined by several parameters of the system [22]. Thus, the $R_{x y}(H)$ should reproduce the field dependence of the magnetization. As shown in Figure $3 \mathrm{a}, R_{\mathrm{S}} \cdot M(H)$ (the Hall resistivity after subtraction of the linear contribution) demonstrates hysteresis behavior (see inset) with $H_{\mathrm{c}} \approx 140$ Oe and a saturation field value of $H_{\mathrm{sat}} \approx 3.8 \mathrm{kOe}$. This is in a good agreement with values of $H_{\mathrm{c}} \approx 130 \mathrm{Oe}$ and $H_{\mathrm{sat}} \approx 4 \mathrm{kOe}$ obtained from SQUID data with the magnetic field oriented perpendicularly to 

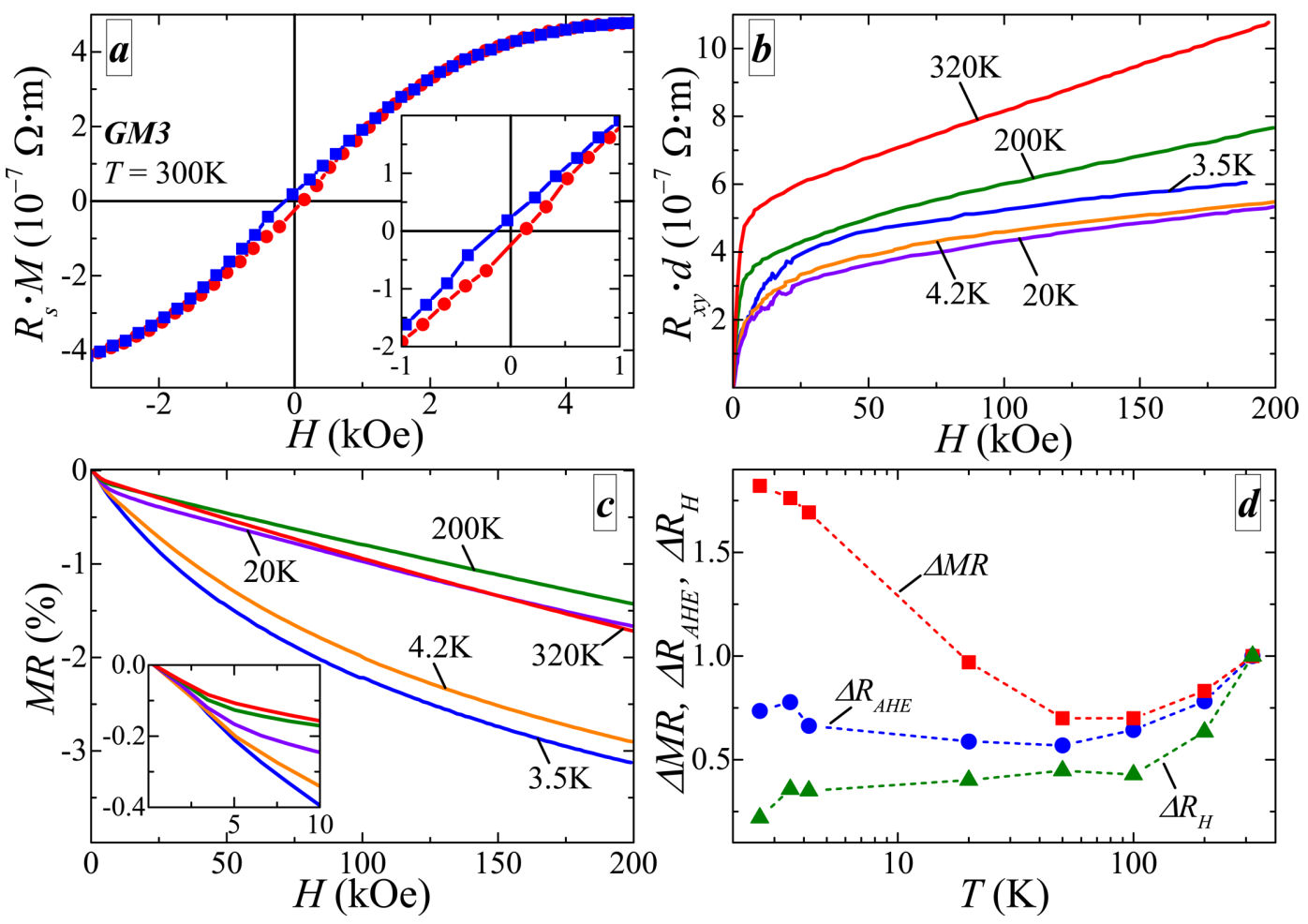

Figure 3: Magnetotransport properties of sample GM3: (a) Field dependence of the anomalous Hall component at $300 \mathrm{~K}$ (the linear background is subtracted). The slope of the curve correlates with the sign of the charge carriers (holes), i.e., the observed AHE is positive. The inset demonstrates hysteresis behaviour at low fields. (b) Field dependence of the Hall resistance up to $H=200 \mathrm{kOe}$ at various temperatures. (c) Magnetoresistance at various temperatures. The inset shows the low-field part of the presented curves. (d) Temperature dependence of the high-field magnetoresistance $\Delta \mathrm{MR}$ (at $H=200 \mathrm{kOe}$ ), saturated AHE amplitude $\Delta R_{\mathrm{AHE}}$ and Hall slope $\Delta R_{\mathrm{H}}$ normalized by the corresponding values at $T=320 \mathrm{~K}$.

the sample plane. Note, that the values of $H_{\mathrm{c}}$ and $H_{\text {sat }}$ in Table 1 were obtained with the magnetic field oriented parallel to the sample plane. The difference between the values in Table 1 and the parameters obtained from the $R_{x y}(H)$ curves is related to a substantial magnetic anisotropy of the samples under study (see Figure 1). The observation of the anomalous Hall effect (AHE) clearly suggests that delocalized holes interact with the magnetic subsystem, i.e., that there are spin-polarized carriers even at room temperature. Thus, the exchange interaction in the studied system can be mediated by these spin-polarized holes as it was suggested earlier.

The combination of the experimentally observed high-temperature ferromagnetism and the spin-polarization of conducting carriers makes the studied films very promising for various applications. In order to obtain a deeper insight into the properties of these films, we have made additional magnetotransport measurements in high magnetic fields. Hall resistivity measurements in magnetic fields up to $H=200 \mathrm{kOe}$ at various temperatures are presented in Figure 3b. It is clearly seen that at room temperature the anomalous Hall effect contribution saturates below $H=10 \mathrm{kOe}$ and $R_{x y}(H)$ becomes linear. The linear slope and the saturation field are different for different temperatures.
To visualise the temperature evolution of the presented curves we used a simple linear fit of the high field region. In this case the fitting function has two variables, the slope $\left(R_{\mathrm{H}}\right)$ and an offset, which corresponds to the saturated AHE amplitude $R_{\mathrm{AHE}}$. A second sign for the interaction between magnetic and conducting subsystems is the appearance of a negative magnetoresistance (nMR), which is usually ascribed to spin-dependent scattering. As it is shown in Figure 3c, the studied samples exhibit nMR that does not saturate up to $200 \mathrm{kOe}$ in the studied temperature range. It should be noted, that at low fields the nMR has a similar form at all temperatures (see inset in Figure 3c), while at higher fields the shape of MR curves changes. In particular, above $20 \mathrm{~K}$ the $\mathrm{nMR}$ is linear above $20 \mathrm{kOe}$, while below $4.2 \mathrm{~K}$ the shape of the nMR is close to sublinear or logarithmic, which is more common for a spin-dependent scattering contribution [23-25].

Based on the assumption of a two-phase magnetic subsystem (MnSb inclusions and isolated Mn atoms) we can qualitatively describe the temperature evolution of the magnetotransport parameters presented in Figure 3d. The character of the dependencies of $\Delta R_{\mathrm{AHE}}$ and $\Delta \mathrm{MR}$ is rather similar. Hence, they should have the same nature. At $50 \mathrm{~K}$ we observe a local minimum in 
both curves. The increase above $50 \mathrm{~K}$ can be related to the presence of Schottky barriers. If both AHE and nMR correspond to the interaction with $\mathrm{MnSb}$ inclusions, then their amplitudes are defined by the tunneling intensity, which increases with temperature (due to the triangular shape of the barrier). Below $50 \mathrm{~K}$ another contribution becomes significant, the interaction with isolated Mn moments. From our data we cannot define the corresponding $T_{\mathrm{c}}$ value accurately because this interaction can be significant even above the ordering temperature [24,25], while the total magnetic moment of isolated $\mathrm{Mn}$ atoms can be substantially smaller than that of $\mathrm{MnSb}$ inclusions. The temperature dependence of $\Delta R_{\mathrm{H}}$ is more complicated. But in the present case of a large AHE contribution, MR and AHE itself can strongly affect $R_{\mathrm{H}}$ through the relations of conductivity and resistivity tensors [26]. It should be noted that nMR at high temperatures can be also due to tensor relations, although the mentioned difference of the nMR functional form at various temperatures suggests that, at least at low temperatures, the nMR should be related to spin-dependent scattering. Also there are several other phenomena that can be relevant. Thus, to elucidate their contributions, a more detailed study with quantitative simulations is needed. However, this it is out of the scope of the present paper.

To establish high-temperature ferromagnetism of $\mathrm{MnSb}$ inclusions via spin-polarized carriers their interaction have to be sufficiently strong. This implies that has to be a large amount of such inclusions with distances between them shorter than the carrier spin-relaxation length. However, the comparatively high carrier mobilities in the studied samples still leave some doubt on the mentioned idea, because a great number of $\mathrm{MnSb}$ inclusions (as well as a high concentration of isolated $\mathrm{Mn}$ atoms) should induce intense scattering of carriers, i.e., low mobilities. The latter, according to estimates, should not exceed $10 \mathrm{~cm}^{2} /(\mathrm{V} \cdot \mathrm{s})$, as it is in GaMnAs [27] and in the previously studied GaMnSb compound with the highest $T_{\mathrm{c}}$ [9]. This contradicts the suggestion that $\mathrm{MnSb}$ nanoinclusions are distributed over the whole volume of the film. Thus, a detailed knowledge of samples structure is required to resolve this problem properly. To get this information we performed additional measurements using S/TEM, AFM and MFM methods.

In the present TEM/EDX microanalysis study of the $\mathrm{GaSb}-\mathrm{MnSb} / \alpha-\mathrm{Al}_{2} \mathrm{O}_{3}$ system the actual investigation was performed on a cross-sectional piece of sample GM3 with lateral sizes of about $1 \mu \mathrm{m}$. The results are presented in Figure $4 \mathrm{a}$ and Figure $4 \mathrm{~b}$. The film thickness is about $150 \mathrm{~nm}$ and the surface roughness does not exceed $6 \mathrm{~nm}$. This is in a good agreement with the data presented in Table 1, which were estimated from the duration of the deposition process. The image analysis confirms a homogeneous composition throughout the film thickness without any substantial contrast variations. Contrast changes in the lateral direction are due to diffraction contrast arising from the columnar film microstructure, which was distinctly observed in bright-field TEM (Figure 4a) and in highresolution bright-field TEM (HRTEM) images (Figure 4b), and even affects the HAADF TEM image (not presented here).

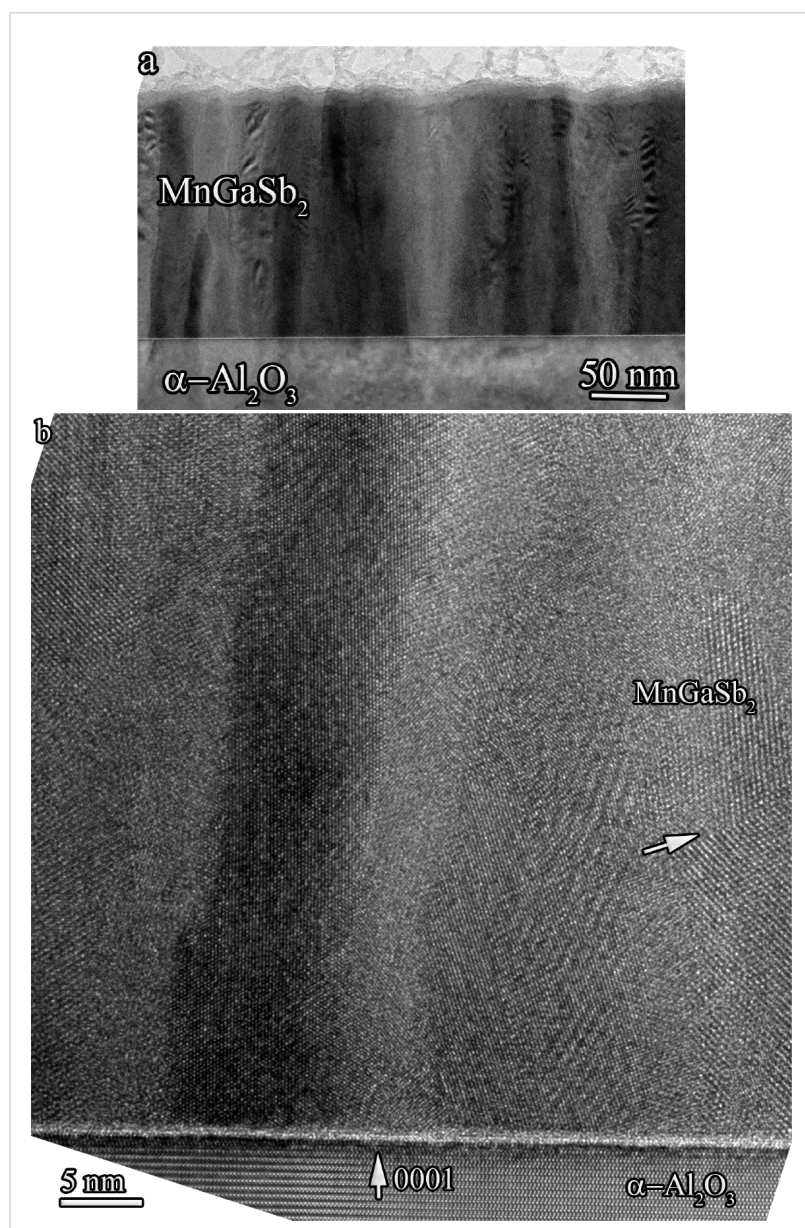

Figure 4: TEM images of the film cross section after annealing (sample GM3): (a) bright-field image, (b) HRTEM image.

Energy-dispersive X-ray microanalysis (EDX) of the film composition near the interface edge and at a distance from it yielded the ratio $\mathrm{Mn} / \mathrm{Ga} / \mathrm{Sb}=30: 30: 40$ with $2 \%$ accuracy. A HRTEM image of studied film is presented in Figure $4 \mathrm{~b}$. Fast Fouriertransform (FFT) analysis of the high-resolution image areas in two directions (Figure 5b and Figure 5e) and direct analysis of the crystal cell image (Figure 5c and Figure 5f) were used to analyze the crystalline structure of the sample. As an example, the results for two of ten studied image areas are presented in Figure 5 .

The analysis of all studied areas unambiguously indicates that the structure of the film is cubic and corresponds to the GaSb 

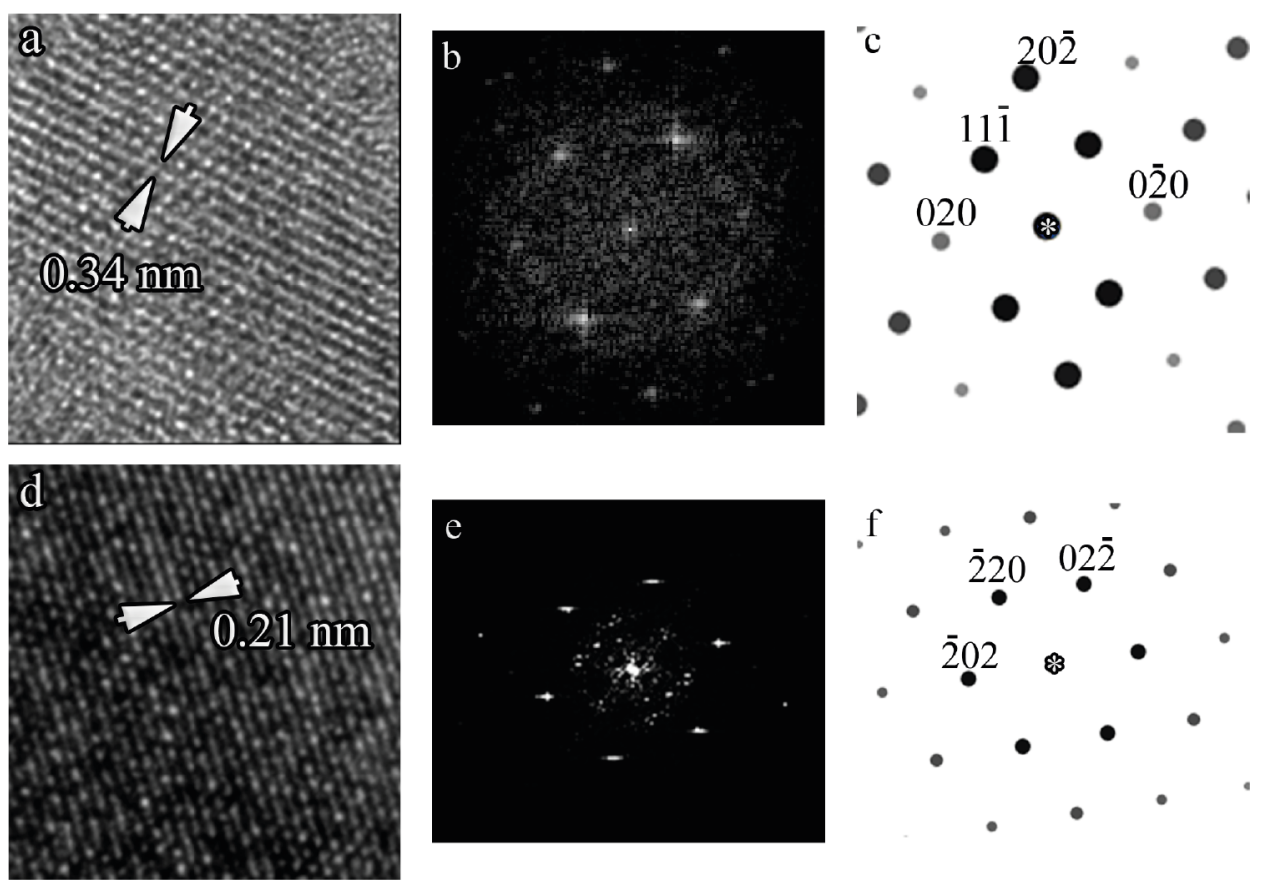

Figure 5: (a,d) HRTEM images of sample areas. (b,e) Corresponding two-dimensional Fourier spectra. (c,f) Calculated electronograms of the cubic $\mathrm{MnGaSb}_{2}$ compound in [101] (c) and [111] (f) projections.

crystal structure (space group $F \overline{4} 3 m$ ). Moreover, the images clearly show twins and stacking faults, which are typcal for cubic GaSb crystals. At the same time, the electron diffraction data obtained for pristine films correspond to diffraction patterns for the hexagonal compound with the space group $P 6_{3} / m m c$. Also, the image analysis showed that the morphology of the film and the lateral dimensions of the film columns remained the same after annealing. It should be noted, that the energy-dispersive X-ray microanalysis showed a slight (2-3\%) decrease of Mn content in the film volume after annealing. This can be related to the details of sample preparation (e.g., the presence of a thicker damaged layer on the surface) or to the diffusion of Mn atoms. Thus, we can conclude that film annealing causes a phase transition of the hexagonal $\mathrm{GaSb}$ matrix to a cubic matrix.

The electron microscopy data unambiguously shows that the columnar microstructure of the film persists up to almost the surface layers. It should be noted, that no signs of second-phase precipitates in the film volume was observed. In particular, using a GaSb-MnSb eutectic composition as a target for sputtering allowed us to avoid the formation of MnGa inclusions, which were observed earlier in samples obtained by laser deposition from $\mathrm{Mn}$ and $\mathrm{GaSb}$ targets, without taking the stoichiometry into account [28].
However, due to unevenness of the film and due to oxidation, the top layer of the film could not be studied carefully and we cannot determine the exact morphology and composition of the surface. Hence, AFM and MFM measurements were performed. AFM and MFM images of one sample are presented in Figure 6. The increase of temperature from 303 to $413 \mathrm{~K}$ does not change surface topology containing regions of larger height (Figure 6a and Figure 6c), while the phase contrast in the MFM images (Figure 6b and Figure 6d), which characterizes magnetization of the film, decreases at higher temperatures. Nevertheless, the MFM images reveal the presence of a considerable phase contrast even at $413 \mathrm{~K}$, which means that the sample exhibits ferromagnetism with $T_{\mathrm{c}}$ much higher than room temperature. As it was mentioned before, high $T_{\mathrm{c}}$ values suggest that observed surface imperfections are MnSb inclusions. A comparison of the AFM and MFM data shows that magnetic moments are distributed mostly within areas of larger height ( $\mathrm{MnSb}$ inclusions) detected by AFM. Magnetic inclusions were detected over the whole film surface. A three-dimensional AFM image of another surface region at $303 \mathrm{~K}$ is shown in Figure 6e. The corresponding MFM image (Figure 6f) demonstrates the presence of sub-micrometer ferromagnetic inclusions with aligned magnetic poles. Combining these results with SEM and TEM data suggests that MnSb inclusions are ferromagnetic and located mostly close to the film surface, rather than being 

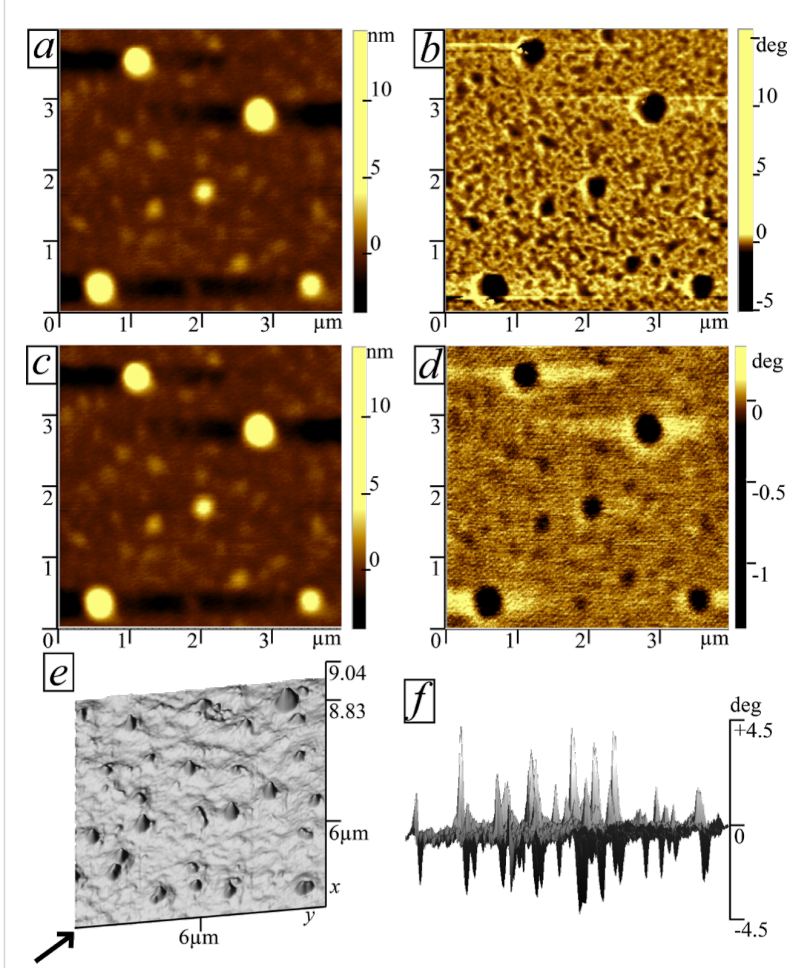

Figure 6: $\operatorname{AFM}(a, c)$ and MFM $(b, d)$ images of the same surface at $303 \mathrm{~K}(\mathrm{a}, \mathrm{b})$ and $413 \mathrm{~K}(\mathrm{c}, \mathrm{d})$. AFM (e) and MFM (f) three-dimensional image of the surface containing ferromagnetic inclusions. The arrow in (e) corresponds to the direction for which MFM image ( $f$ ) was made.

evenly distributed within the sample volume. This assumption also provides a good explanation for the high mobility values of conducting holes, since magnetic inclusions localized on the surface have a substantially weaker effect on scattering in studied films.

\section{Conclusion}

We have studied structural, magnetic and magnetotransport properties of annealed $(\mathrm{GaSb})_{1-x}(\mathrm{MnSb})_{x}$ films with $x=0.41$ and thickness $d=120-135 \mathrm{~nm}$. Electron microscopy data suggests that studied films have single-phase columnar structure in the volume. It was found that annealing process induces a phase transition of hexagonal $\mathrm{GaSb}$ matrix into a cubic matrix. AFM and MFM studies revealed the presence of ferromagnetic $\mathrm{MnSb}$ inclusions near the surface of the films. This perfectly explains the high mobility values of charge carriers in these systems. Experimental observation of these inclusions is crucial for the explanation of high-temperature ferromagnetism, since the magnetization hysteresis at room temperature perfectly fits the idea of a dominant contribution of MnSb inclusions with $T_{\mathrm{c}}>400 \mathrm{~K}$ interacting with holes. This is confirmed by the appearance of a substantial anomalous Hall effect even at room temperature that signifies the presence of spin-polarized charge carriers. The interaction of charge carriers with inclusions is greatly affected by the Schottky barriers at $\mathrm{GaSb} / \mathrm{MnSb}$ boundaries. Due to high carrier concentrations in the studied films, the transparency of these barriers is rather high, which explains the saturation of magnetization at $N_{\mathrm{p}}=10^{20} \mathrm{~cm}^{-3}$.

\section{Acknowledgements}

This work was partially supported by Russian Foundation for Basic Research (grants \#17-02-00262 and \#16-03-00150) and by Ministry of Education and Science of Russian Federation (grant \#16.2814.2017/PCh).

\section{ORCID ${ }^{\circledR}$ iDs}

Alexey V. Kochura - https://orcid.org/0000-0002-7941-8404 Alexander L. Vasiliev - https://orcid.org/0000-0001-7884-4180

\section{References}

1. Jungwirth, T.; Sinova, J.; Mašek, J.; Kučera, J.; MacDonald, A. H. Rev. Mod. Phys. 2006, 78, 809-864. doi:10.1103/revmodphys.78.809

2. Dietl, T. Semiconductor Spintronics. In Modern Aspects of Spin Physics; Pötz, W.; Hohenester, U.; Fabian, J., Eds.; Springer: Berlin, Germany, 2007; pp 1-46. doi:10.1007/3-540-38592-4_1

3. Dietl, T.; Ohno, H. Rev. Mod. Phys. 2014, 86, 187-251. doi:10.1103/revmodphys.86.187

4. Chen, L.; Yan, S.; Xu, P. F.; Lu, J.; Wang, W. Z.; Deng, J. J.; Qian, X.; Ji, Y.; Zhao, J. H. Appl. Phys. Lett. 2009, 95, 182505. doi:10.1063/1.3259821

5. Wang, M.; Marshall, R. A.; Edmonds, K. W.; Rushforth, A. W.; Campion, R. P.; Gallagher, B. L. Appl. Phys. Lett. 2014, 104, 132406. doi:10.1063/1.4870521

6. Chen, L.; Yang, X.; Yang, F.; Zhao, J.; Misuraca, J.; Xiong, P.; von Molnár, S. Nano Lett. 2011, 11, 2584-2589. doi:10.1021/nl201187m

7. Lawniczak-Jablonska, K.; Libera, J.; Wolska, A.; Klepka, M. T.; Dluzewski, P.; Sadowski, J.; Wasik, D.; Twardowski, A.; Kwiatkowski, A.; Sato, K. Phys. Status Solidi RRL 2011, 5, 62-64. doi:10.1002/pssr.201004503

8. Lawniczak-Jablonska, K.; Wolska, A.; Klepka, M. T.; Kret, S.; Gosk, J.; Twardowski, A.; Wasik, D.; Kwiatkowski, A.; Kurowska, B.; Kowalski, B. J.; Sadowski, J. J. Appl. Phys. 2011, 109, 074308. doi:10.1063/1.3562171

9. Rylkov, V. V.; Aronzon, B. A.; Danilov, Yu. A.; Drozdov, Yu. N.; Lesnikov, V. P.; Maslakov, K. I.; Podol'skii, V. V. J. Exp. Theor. Phys. 2005, 100, 742-751. doi:10.1134/1.1926435

10. Braun, W.; Trampert, A.; Kaganer, V. M.; Jenichen, B.; Satapathy, D. K.; Ploog, K. H. J. Cryst. Growth 2007, 301-302, 50-53. doi:10.1016/j.jcrysgro.2006.09.022

11. Marenkin, S. F.; Novodvorsky, O. A.; Shorokhova, A. V.; Davydov, A. B.; Aronzon, B. A.; Kochura, A. V.; Fedorchenko, I. V.; Khramova, O. D.; Timofeev, A. V. Inorg. Mater. 2014, 50, 897-902. doi:10.1134/s0020168514090076

12. Koplak, O. V.; Polyakov, A. A.; Davydov, A. B.; Morgunov, R. B.; Talantsev, A. D.; Kochura, A. V.; Fedorchenko, I. V.; Novodvorskii, O. A.; Parshina, L. S.; Khramova, O. D.; Shorokhova, A. V.; Aronzon, B. A. J. Exp. Theor. Phys. 2015, 120 , 1012-1018. doi:10.1134/s1063776115050131

13. Talantsev, A.; Koplak, O.; Morgunov, R. Superlattices Microstruct. 2016, 95, 14-23. doi:10.1016/j.spmi.2016.04.012 
14. Matsukura, F.; Abe, E.; Ohno, H. J. Appl. Phys. 2000, 87, 6442-6444. doi:10.1063/1.372732

15. Lotin, A. A.; Novodvorsky, O. A.; Khaydukov, E. V.; Glebov, V. N.; Rocheva, V. V.; Khramova, O. D.; Panchenko, V. Y.; Wenzel, C.; Trumpaicka, N.; Chtcherbachev, K. D. Semiconductors 2010, 44, 246-250. doi:10.1134/s106378261002020x

16. Stadelmann, P. A. Ultramicroscopy 1987, 21, 131-145. doi:10.1016/0304-3991(87)90080-5

17. Novotortsev, V. M.; Kochura, A. V.; Marenkin, S. F.; Fedorchenko, I. V.; Drogunov, S. V.; Lashkul, A.; Lähderanta, E. Russ. J. Inorg. Chem. 2011, 56, 1951-1956. doi:10.1134/s0036023611120400

18. Teramoto, I.; Van Run, A. J. Phys. Chem. Solids 1968, 29, 347-355. doi:10.1016/0022-3697(68)90080-2

19. Yakovleva, E. I.; Oveshnikov, L. N.; Kochura, A. V.; Lisunov, K. G.; Lahderanta, E.; Aronzon, B. A. JETP Lett. 2015, 101, 130-135. doi:10.1134/s0021364015020149

20. Coehoorn, R.; Haas, C.; de Groot, R. A. Phys. Rev. B 1985, 31 , 1980-1996. doi:10.1103/physrevb.31.1980

21. Sze, S. M. Physics of Semiconductor Devices, 2nd ed.; Wiley-Interscience: New York, NY, U.S.A., 1981.

22. Nagaosa, N.; Sinova, J.; Onoda, S.; MacDonald, A. H.; Ong, N. P. Rev. Mod. Phys. 2010, 82, 1539-1592. doi:10.1103/revmodphys.82.1539

23. Xiao, J. Q.; Jiang, J. S.; Chien, C. L. Phys. Rev. Lett. 1992, 68, 3749-3752. doi:10.1103/physrevlett.68.3749

24. Oveshnikov, L. N.; Kulbachinskii, V. A.; Davydov, A. B.; Aronzon, B. A.; Rozhansky, I. V.; Averkiev, N. S.; Kugel, K. I.; Tripathi, V. Sci. Rep. 2015, 5, 17158. doi:10.1038/srep17158

25. Oveshnikov, L. N.; Nekhaeva, E. I. Semiconductors 2017, 51, 1313-1320. doi:10.1134/s1063782617100177

26. Shen, S.; Liu, X.; Ge, Z.; Furdyna, J. K.; Dobrowolska, M.; Jaroszynski, J. J. Appl. Phys. 2008, 103, 07D134. doi:10.1063/1.2838477

27. Hayashi, T.; Tanaka, M.; Nishinaga, T.; Shimada, H. J. Appl. Phys. 1997, 81, 4865-4867. doi:10.1063/1.364859

28. Bobrov, A. I.; Pavlova, E. D.; Kudrin, A. V.; Malekhonova, N. V. Semiconductors 2013, 47, 1587-1590. doi:10.1134/s1063782613120038

\section{License and Terms}

This is an Open Access article under the terms of the Creative Commons Attribution License (http://creativecommons.org/licenses/by/4.0). Please note that the reuse, redistribution and reproduction in particular requires that the authors and source are credited.

The license is subject to the Beilstein Journal of Nanotechnology terms and conditions: (https://www.beilstein-journals.org/bjnano)

The definitive version of this article is the electronic one which can be found at: doi:10.3762/bjnano.9.230 